\title{
TENSOR PRODUCTS AND ALMOST PERIODICITY ${ }^{1}$
}

\author{
HUGO D. JUNGHENN
}

\begin{abstract}
Let $E$ and $F$ be locally convex spaces and $G$ their completed $\varepsilon$-tensor product. It is shown that if $S$ and $T$ are weakly almost periodic equicontinuous semigroups of operators on $E$ and $F$ respectively, then, under mild restrictions on $E$ or $F, S \otimes T$ is a weakly almost periodic equicontinuous semigroup of operators on $G$, and the almost periodic and flight vector subspaces of $G$ are related in a natural way to the corresponding subspaces of $E$ and $F$ via the $\varepsilon$-tensor product. Furthermore, if $E$ and $F$ both decompose into a direct sum of these subspaces then so does $G$.
\end{abstract}

1. Weakly almost periodic semigroups. Let $E$ be a locally convex (Hausdorff) linear topological space with topological dual $E^{\prime}$, and let $L(E)$ denote the space of continuous linear operators on $E$. A semigroup of operators on $E$ is a subset $S$ of $L(E)$ containing the identity operator and closed under composition. A vector $x \in E$ is said to be weakly (strongly) almost periodic under $S$ if its orbit $S x=\{u x: u \in S\}$ is relatively compact in the weak (strong) topology of $E$. The set of all weakly (strongly) almost periodic vectors in $E$ shall be denoted by $W(E, S)(A(E, S))$. Occasionally we shall suppress the symbols $E$ or $S$ from the notation if they are understood from context. If $W(E, S)=E(A(E, S)=E)$ we say that $S$ is weakly (strongly) almost periodic.

It is easily seen that multiplication in a semigroup of operators $S$ on a locally convex space $E$ is separately continuous with respect to the weak or strong operator topologies on $L(E)$, that is to say $S$ is a topological semigroup. Moreover if $S$ is equicontinuous then multiplication is actually jointly continuous in the strong operator topology. The following lemma is at the heart of the theory of weak almost periodicity. A proof can be found in [1].

Lemma 1.1. Let $S$ be a weakly almost periodic equicontinuous semigroup of operators on a locally convex space $E$, and let $S$ denote the closure

Received by the editors March 26, 1973 and, in revised form June 21, 1973.

AMS (MOS) subject classifications (1970). Primary 47D05, 46M05; Secondary 43A60, $22 \mathrm{~A} 15$.

Key words and phrases. Semigroup of operators, $\varepsilon$-tensor product, weakly almost periodic, almost periodic, flight vector, reversible vector.

${ }^{1}$ Research supported by the National Science Foundation (under Institutional Grant GU 3287 to George Washington University). 
of $S$ in the weak operator topology of $L(E)$. Then $S$ is a compact topological semigroup in the weak operator topology.

In connection with Lemma 1.1 we remark that if $E$ is barreled or a Baire space then the weak almost periodicity of $S$ implies equicontinuity [8, p. 83], and if $E$ is semireflexive then the converse implication holds $[8$, p. 144].

Using Lemma 1.1 the following generalization of a result of Eberlein [3] is easily proved (see [1]).

THEOREM 1.2. Let $S$ be an equicontinuous semigroup of operators on a locally convex space $E$. Then $W=W(E, S)$ is an S-invariant linear subspace of $E$. Moreover, if $E$ is complete then $W$ is closed.

COROLlaRY 1.3. If $S$ is a weakly almost periodic equicontinuous semigroup on $E$, then the extension of $S$ to the completion of $E$ is a weakly almost periodic equicontinuous semigroup.

2. Tensor product of weakly almost periodic semigroups. Let $E$ and $F$ be locally convex spaces and $G=E \otimes_{\varepsilon} F$ the completion in the $\varepsilon$ topology of the tensor product $E \otimes F$. If $S$ and $T$ are semigroups of operators on $E$ and $F$ respectively we shall let $S \otimes T$ denote the set of all operators in $L(G)$ of the form $u \otimes v$, where $u \in S$ and $v \in T$. Recall that $u \otimes v$ is defined by the equation $(u \otimes v)(x \otimes y)=u x \otimes v y$. It follows easily that $S \otimes T$ is a semigroup of operators on $G$, where $\left(u_{1} \otimes v_{1}\right)\left(u_{2} \otimes v_{2}\right)=$ $u_{1} u_{2} \otimes v_{1} v_{2}$. Furthermore, if $S$ and $T$ are equicontinuous then so is $S \otimes T$ [5].

Our main object in this section is to determine conditions on $E$ and $F$ under which the weak almost periodicity of $S$ and $T$ implies that of $S \otimes T$. To this end we require the following lemma.

Lemma 2.1. Let $E$ and $F$ be locally convex spaces, $A$ and $B$ relatively weakly compact subsets of $E$ and $F$ respectively. Suppose one of the following conditions holds:

(i) E or F has separable dual;

(ii) $E$ or $F$ is a Banach space;

(iii) $A$ or $B$ is relatively strongly compact.

Then $A \otimes B=\{x \otimes y: x \in A, y \in B\}$ is relatively compact in the weak topology of $G=E \otimes_{\varepsilon} F$.

Proof. By the completeness of $G$ it suffices to show that any sequence $\left(x_{n} \otimes y_{n}\right)$ in $A \otimes B$ has a weak cluster point [8, p. 187]. Let $\left(x_{\alpha}\right)$ be a subnet of $\left(x_{n}\right)$ converging weakly to $x_{0} \in E$ and $\left(y_{\alpha}\right)$ a subnet of $\left(y_{n}\right)$ converging weakly to $y_{0} \in F$. We shall show that $x_{\alpha} \otimes y_{\alpha}$ converges weakly to $x_{0} \otimes y_{0}$ in $G$. The equality $x_{\alpha} \otimes y_{\alpha}-x_{0} \otimes y_{0}=\left(x_{\alpha}-x_{0}\right) \otimes y_{0}+x_{\alpha} \otimes\left(y_{\alpha}-y_{0}\right)$ and the 
separate weak continuity of tensor product show that it suffices to prove that $x_{\alpha} \otimes\left(y_{\alpha}-y_{0}\right)$ converges weakly to zero. Let $\varphi \in G^{\prime}$ and suppose $\dot{\varphi}\left(x_{\alpha} \otimes\left(y_{\alpha}-y_{0}\right)\right)$ does not converge to zero. Then there exist a positive number $\varepsilon$ and a subnet $x_{\beta} \otimes\left(y_{\beta}-y_{0}\right)$ such that $\left|\varphi\left(x_{\beta} \otimes\left(y_{\beta}-y_{0}\right)\right)\right| \geqq \varepsilon$ for all $\beta$. Now for all $x \in E, y \in F$,

$$
\varphi(x \otimes y)=\int_{A^{\prime} \times B^{\prime}}\left\langle x, x^{\prime}\right\rangle\left\langle y, y^{\prime}\right\rangle d \mu\left(x^{\prime}, y^{\prime}\right)
$$

where $A^{\prime}$ and $B^{\prime}$ are equicontinuous subsets of $E^{\prime}$ and $F^{\prime}$ respectively and $\mu$ is a Borel measure on $A^{\prime} \times B^{\prime}$ with total variation $|\mu| \leqq 1[8$, p. 168]. Since $A$ is bounded, $\lambda=\sup \left\{\left|\left\langle x, x^{\prime}\right\rangle\right|: x \in A, x^{\prime} \in A^{\prime}\right\}<\infty$. Therefore for all $\beta$ we have

$$
\int_{A^{\prime} \times B^{\prime}}\left|\left\langle y_{\beta}-y_{0}, y^{\prime}\right\rangle\right| d|\mu|\left(x^{\prime}, y^{\prime}\right) \geqq \varepsilon / \lambda>0 .
$$

Suppose $F^{\prime}$ is separable with total set $\left\{y_{n}^{\prime}\right\}$, and choose a sequence $\left(y_{k}\right)$ from the set $\left\{y_{\beta}\right\}$ such that $\lim _{k \rightarrow \infty}\left\langle y_{k}-y_{0}, y_{n}^{\prime}\right\rangle=0$ for every $n$. Since $B$ is bounded, it follows easily that $y_{k}$ converges weakly to $y_{0}$. By Lebesgue's Dominated Convergence Theorem we thus obtain a contradiction to (1).

Now assume $F$ is a Banach space. Then by Eberlein's Theorem we may suppose $y_{n}$ converges weakly to $y_{0}$ and in the same manner as above we contradict (1).

Finally, if $B$ is relatively strongly compact then we may assume $\left\langle y_{\beta}-y_{0}, y^{\prime}\right\rangle$ converges to 0 uniformly on $\boldsymbol{B}^{\prime}$ and again we contradict (1). Q.E.D.

We may now state and prove the main result of this section.

THEOREM 2.2. Let $E$ and $F$ be locally convex spaces, $S$ and $T$ weakly almost periodic equicontinuous semigroups of operators on $E$ and $F$ respectively, and let $G=E \otimes_{\varepsilon} F$. Suppose further that one of the following conditions holds:

(i) E or $F$ has separable dual;

(ii) $E$ or $F$ is a Banach space;

(iii) $S$ or $T$ is strongly almost periodic.

Then $S \otimes T$ is a weakly almost periodic equicontinuous semigroup of operators on $G$, and $\mathrm{Cl}(S \otimes T)=S \otimes T$ (closures taken in the weak operator topologies).

Proof. If $x \in E$ and $y \in F$, then $S \otimes T(x \otimes y)=S x \otimes T y$; hence if any of the conditions (i)-(iii) holds, Lemma 2.1 implies that $x \otimes y \in W(G, S \otimes T)$. By Theorem 1.2, $G \subset W(G)$, i.e., $S \otimes T$ is weakly almost periodic.

Let $w \in \mathrm{Cl}(S \otimes T),\left(u_{\alpha}\right)$ and $\left(v_{\alpha}\right)$ nets in $S$ and $T$ respectively such that $u_{\alpha} \otimes v_{\alpha}$ converges to $w$ in the weak operator topology of $L(G)$. We may 
assume $u_{\alpha}$ converges to $u \in S$ and $v_{\alpha}$ converges to $v \in \bar{T}$. Let $x \in E, y \in F$, $x^{\prime} \in E^{\prime}, y^{\prime} \in F^{\prime}$. Then $x^{\prime} \otimes y^{\prime}\left(u_{\alpha} \otimes v_{\alpha}(x \otimes y)\right)=\left\langle u_{\alpha} x, x^{\prime}\right\rangle\left\langle v_{\alpha} y, y^{\prime}\right\rangle$ converges to $\left\langle u x, x^{\prime}\right\rangle\left\langle v y, y^{\prime}\right\rangle$, hence $x^{\prime} \otimes y^{\prime}(u \otimes v(x \otimes y)-w(x \otimes y))=0$. If $\varphi \in G^{\prime}$, then by definition of the $\varepsilon$-topology $|\varphi(\theta)| \leqq \sup \left\{\left|x^{\prime} \otimes y^{\prime}(\theta)\right|: x^{\prime} \in A^{\prime}, y^{\prime} \in B^{\prime}\right\}$ for all $\theta \in G$, where $A^{\prime}$ and $B^{\prime}$ are equicontinuous subsets of $E^{\prime}$ and $F^{\prime}$ respectively [5]. It follows that $\varphi(u \otimes v(x \otimes y)-w(x \otimes y))=0$ and hence that $w=u \otimes v \in \bar{S} \otimes \bar{T}$.

Conversely, let $u \otimes v \in S \otimes \bar{T}$ and let $\left(u_{\alpha}\right)$ and $\left(v_{\alpha}\right)$ be as before. For fixed $\beta, u \otimes v_{\beta}$ is the weak operator limit of $u_{\alpha} \otimes v_{\beta}$ and is therefore a member of $\mathrm{Cl}(S \otimes T)$. Taking the limit with respect to $\beta$ we see that $u \otimes v \in \mathrm{Cl}(S \otimes T)$. Q.E.D.

3. Decomposition of $E \otimes_{c} F$. In this section we shall determine conditions under which $E \otimes_{\varepsilon} F$ has a direct sum decomposition into subspaces of almost periodic and flight vectors.

If $S$ is a weakly almost periodic semigroup of operators on the locally convex space $E$, we denote by $E_{r}\left(E_{0}\right)$ the set of all vectors $x \in E$ having the property that $\mathrm{Cl}(S x)=\mathrm{Cl}(S y)$ for all $y \in \mathrm{Cl}(S x)(0 \in \mathrm{Cl}(S x))$, where the closures are in the weak topology of $E . E_{r}$ is the set of reversible vectors of $E, E_{0}$ the set of flight vectors [6]. Also, we shall let $E_{p}$ denote the closed linear span of all finite-dimensional $S$-invariant subspaces $H$ of $E$ which have the property that $S$ restricted to $H$ is contained in an equicontinuous (i.e., uniformly bounded) group of operators on $H . E_{p}$ is the set of almost periodic vectors [2].

The proofs of the following theorems rely heavily on the ideal theory of compact topological semigroups as developed by deLeeuw and Glicksberg in [2]. In particular we shall make use of the fact that a compact topological semigroup $R$ contains a smallest (nonempty) two-sided ideal $K(R)$, called the kernel of $R$, and that $K(R)$ contains at least one idempotent element.

We shall also need the analogs of Theorems 4.9, 4.10 and 4.11 of [2] in the setting of locally convex spaces. An examination of the proofs of these theorems reveals the following: Theorem 4.9 holds for any locally convex space and Theorems $4.10,4.11$ hold for quasi-complete spaces. For the details the interested reader is referred to [7]. These theorems may also be formulated so that no reference to topology need be made [1].

THEOREM 3.1. Let $S$ and $T$ be weakly almost periodic equicontinuous semigroups of operators on the locally convex spaces $E$ and $F$ respectively. If $S \otimes T$ is weakly almost periodic on $G=E \otimes_{\varepsilon} F$ and if $E_{0}$ and $F_{0}$ are closed invariant linear spaces, then $G_{0}$ is a closed invariant linear subspace of $G$ and is the closure of $E_{0} \otimes F+E \otimes F_{0}$. 
Proof. By Theorem 4.9 of [2] $S$ and $\bar{T}$ have unique minimal left ideals $I$ and $J$ respectively. To show $G_{0}$ is a closed invariant subspace of $G$ it suffices by the same theorem to show that $\operatorname{Cl}(S \otimes T)(=S \otimes \bar{T})$ has a unique minimal left ideal, namely $I \otimes J$.

It is clear that $I \otimes J$ is a left ideal of $\mathrm{Cl}(S \otimes T)$. To show that it is minimal let $K$ be a left ideal of $\mathrm{Cl}(S \otimes T)$ contained in $I \otimes J$, and choose any $u_{0} \otimes$ $v_{0} \in K$ such that $u_{0} \in I, v_{0} \in J$. Now, by Corollary 2.4 of [2], $I=K(\bar{S})$, and by Theorem 2.3 [2], $I u_{0}=I$. Hence if $e$ is any projection in $K(S)$, then there exists $u \in I$ such that $u u_{0}=e$. Similarly, if $f$ is a projection in $K(\bar{T})$, there exists $v \in J$ such that $v v_{0}=f$. Since $K$ is a left ideal, $e \otimes f=(u \otimes v)\left(u_{0} \otimes v_{0}\right) \in K$. Fix $e$ and $f$ and let $I_{1}=\{u \in I: u \otimes f \in K\} . I_{1}$ is easily seen to be a nonempty left ideal of $S$, hence $I_{1}=I$, i.e., $u \otimes f \in K$ for every $u \in I$. Now let $J_{1}=\{v \in J: e u \otimes v \in K\}$, where $u$ is a fixed element of $I_{\text {. }} J_{1}$ is a left ideal of $J$, and, by what has just been proved, $J_{1}$ contains $f$. Therefore $J_{1}=J$, and we have shown that $e u \otimes v \in K$ for all $u \in I, v \in J$ and all projections $e \in I$. By Corollary 2.4 of [2], $I$ is the union of all right ideals $e \bar{S}$, where $e^{2}=e \in I$. Hence given any $u \in I$ there exists a projection $e \in I$ such that $e u=u$, and it follows from above that $I \otimes J=K$.

By Theorem 2.3 of [2], $K(\mathrm{Cl}(S \otimes T))$ is the union of all minimal left ideals of $\mathrm{Cl}(S \otimes T)$ and therefore contains $I \otimes J=K(\bar{S}) \otimes K(\bar{T})$. But the latter is a two-sided ideal and so must equal $K(\mathrm{Cl}(S \otimes T))$. Thus $I \otimes J$ contains all minimal left ideals and therefore must be the unique minimal left ideal of $\mathrm{Cl}(S \otimes T)$.

Now let $\theta=\lim _{\alpha} \theta_{\alpha} \in G_{0}$, where $\left(\theta_{\alpha}\right)$ is a net in $E \otimes F$. By Lemma 4.2 of [2] there exists a projection $g \in K(\mathrm{Cl}(S \otimes T))=K(\bar{S}) \otimes K(\bar{T})$ such that $g(\theta)=0$. Let $e \in K(\bar{S})$ and $f \in K(\bar{T})$ be arbitrary projections. Then $e \otimes f$ is a projection in $K(\mathrm{Cl}(S \otimes T))$, hence $(e \otimes f) g=e \otimes f$ by Corollary 2.4 [2]. In particular, $e \otimes f(\theta)=0$, hence $\theta=\lim _{\alpha}\left(\theta_{\alpha}-e \otimes f\left(\theta_{\alpha}\right)\right)$. For a fixed $\theta_{\alpha}=\sum_{i=1}^{n} x_{i} \otimes y_{i}$,

$$
\begin{aligned}
\theta_{\alpha}-e \otimes f\left(\theta_{\alpha}\right)= & \sum\left(x_{i}-e x_{i}\right) \otimes y_{i} \\
& +\sum e x_{i} \otimes\left(y_{i}-f y_{i}\right) \in E_{0} \otimes F+E \otimes F_{0},
\end{aligned}
$$

so $\theta \in \mathrm{Cl}\left(E_{0} \otimes F+E \otimes F_{0}\right)$. Therefore we have $G_{0} \subset \mathrm{Cl}\left(E_{0} \otimes F+E \otimes F_{0}\right)$. The reverse inclusion follows readily from the fact that $G_{0}$ is a closed subspace of $G$. Q.E.D.

THEOREM 3.2. Let $S$ and $T$ be weakly almost periodic equicontinuous semigroups of operators on the quasi-complete locally convex spaces $E$ and $F$ respectively, and suppose $S \otimes T$ is weakly almost periodic on $G=E \otimes_{\varepsilon} F$. If $E_{r}=E_{p}$ and $F_{r}=F_{p}$, then $G_{r}=G_{p}=E_{p} \otimes_{\varepsilon} F_{p}$.

Proof. The hypotheses imply that $\bar{S}$ and $\bar{T}$ have unique minimal right ideals $I$ and $J$ respectively [2, Theorem 4.10]. By methods analogous 


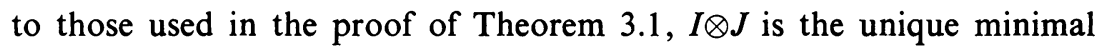
right ideal of $\mathrm{Cl}(S \otimes T)$. Hence by Theorem 4.10 [2], $G_{r}=G_{p}$.

If $x \in E_{p}$ and $y \in F_{p}$, there exist projections $e \in K(\bar{S}), f \in K(\bar{T})$ such that $e x=x$ and $f y=y$ [2, Lemma 4.1]. Then $e \otimes f$ is a projection in $K(\mathrm{Cl}(S \otimes T))=K(\bar{S}) \otimes K(\bar{T})$, and the same lemma shows that $x \otimes y \in G_{p}$. Thus $E_{p} \otimes_{\varepsilon} F_{p} \subset G_{p}$. Conversely, let $\theta=\lim _{\alpha} \theta_{\alpha} \in G_{p}, \theta_{\alpha} \in E \otimes F$. Choose a projection $g \in K(\mathrm{Cl}(S \otimes T))$ such that $g \theta=\theta$. If $e$ and $f$ are arbitrary projections in $K(\bar{S})$ and $K(\bar{T})$ respectively, then $e \otimes f$ is a projection in $K(\mathrm{Cl}(S \otimes T))$ and by Corollary 2.4 [2], $(e \otimes f) g=g$. It follows that $\theta=e \otimes f(\theta)=\lim _{\alpha} e \otimes f\left(\theta_{\alpha}\right)$. If $\theta_{\alpha}=\sum_{i=1}^{n} x_{i} \otimes y_{i}$, then $e \otimes f\left(\theta_{\alpha}\right)=\sum e x_{i} \otimes f y_{i} \in$ $E_{p} \otimes F_{p}$, hence $\theta \in E_{p} \otimes_{\varepsilon} F_{p}$. Therefore $G_{p}=E_{p} \otimes_{\varepsilon} F_{p}$. Q.E.D.

We may now prove the main result of this section.

THEOREM 3.3. If all the hypotheses of Theorems 3.1 and 3.2 are satisfied, then $G=G_{p} \oplus G_{0}$, where $G_{p}=E_{p} \otimes_{\varepsilon} F_{p}$ and $G_{0}=\mathrm{Cl}\left(E_{0} \otimes F+E \otimes F_{0}\right)$.

Proof. By Theorem 4.11 [2], $K(\bar{S})$ and $K(\bar{T})$ are compact topological groups; to show $G=G_{p} \oplus G_{0}$ it suffices by the same theorem to show that $K(\mathrm{Cl}(S \otimes T))$ is a compact topological group. By Ellis' Theorem [4] we need only show that $K(\mathrm{Cl}(S \otimes T))$ is algebraically a group. But this is immediate from the equality $K(\mathrm{Cl}(S \otimes T))=K(S) \otimes K(\bar{T})$ (see proof of Theorem 3.1). Q.E.D.

The above results may be used in a variety of ways to generate nontrivial examples of weakly almost periodic semigroups of operators with the decomposition property of Theorem 3.3. As an illustration, let $E$ and $F$ be reflexive Banach spaces and let $S$ and $T$ be bounded. Then $S$ and $T$ are obviously weakly almost periodic, hence, according to Theorem 2.2, so is $S \otimes T$. Since $G=E \otimes_{\varepsilon} F$ need not be reflexive [9], this result is decidedly nontrivial. Furthermore, if, say, $E$ and $E^{\prime}$ are strictly convex and $T$ is commutative, then $E$ and $F$ both have direct sum decompositions into almost periodic and flight vector subspaces [2], and therefore, by Theorem 3.3 , so does $G$.

\section{REFERENCES}

1. J. Berglund and $\mathrm{K}$. Hofmann, Compact semitopological semigroups and weakly almost periodic functions, Lecture Notes in Math., no. 42, Springer-Verlag, New York and Berlin, 1967. MR 36 \#6531.

2. K. deLeeuw and I. Glicksberg, Applications of almost periodic compactifications, Acta Math. 105 (1961), 63-97. MR 24 \#A1632.

3. W. F. Eberlain, Abstract ergodic theorems and weak almost periodic functions, Trans. Amer. Math. Soc. 67 (1949), 217-240. MR 12, 112.

4. R. Ellis, Locally compact transformation groups, Duke Math. J. 24 (1957), 119-125. MR 19, 561.

5. A. Grothendieck, Produits tensoriels topologiques et espaces nucléaires, Mem. Amer. Math. Soc. No. 16 (1955). MR 17, 763. 
6. K. Jacobs, Ergodentheorie und fastperiodische Functionen auf Halbgruppen, Math. Z. 64 (1956), 298-338. MR 17, 988.

7. H. Junghenn, Almost periodic compactifications and applications to one-parameter semigroups, Thesis, The George Washington University, Washington, D.C., 1971.

8. H. H. Schaefer, Topological vector spaces, Graduate Texts in Math., SpringerVerlag, New York, 1971.

9. R. Schatten, A theory of cross-spaces, Ann. of Math. Studies, no. 26, Princeton Univ. Press, Princeton, N.J., 1950. MR 12, 186.

Department of Mathematics, George Washington University, Washington, D.C. 20006 